\title{
Hierarchical Reinforcement of Randomly-Oriented Carbon Nanotube Mats by Ion Irradiation
}

Francesco Fornasiero, ${ }^{\text {a, }}$ Mary LeBlanc, ${ }^{\text {a }}$ Supakit Charnvanichborikarn, ${ }^{\text {a }}$ Sergei O. Kucheyev, ${ }^{\text {a }}$ Swanee J. Shin, ${ }^{a}$ Kuanping Gong, ${ }^{\mathrm{b}}$ Lijie Ci, ${ }^{\mathrm{c}}$ Jinseong Park, ${ }^{\mathrm{b}}$ and Robin Miles ${ }^{\mathrm{a}}$

Affiliations:

${ }^{a}$ Lawrence Livermore National Laboratory, 7000 East Avenue, Livermore CA 94550, USA

b San Jose Lab, Samsung Cheil Industries, 2186 Bering Dr. San Jose, CA 95131, USA

c School of Materials Science and Engineering, Shandong University, Jinan 250061, China

\begin{abstract}
Because of their outstanding mechanical properties, carbon nanotubes (CNTs) are attractive 1-D nanoscale building blocks for lightweight composites that could potentially outperform naturally occurring materials. A still unsolved challenge for fully exploiting CNT superior mechanical properties for reinforcement of macroscopic systems is control of the interfacial interaction across multiple length scales to favor load transfer. Toward overcoming this challenge, we investigate here the effect of high-energy $\mathrm{He}^{+}$ion irradiation on the elastic modulus and tensile strength of thin films of randomly oriented double-walled nanotube (DWCNT) mats and DWCNT-epoxy composites. We correlate irradiation-induced reinforcement with the formation of cross-links at different hierarchical levels of the DWCNT network. Our measurements reveal a rapidly increasing reinforcement at low ion doses, attributed to intra-bundle CNT cross-links, followed by a slow mechanical reinforcement at higher doses, associated to inter-bundle cross-links. At the highest ion irradiation dose of this study, the strength and elastic modulus of DWCNT-epoxy composites approach $\sim 900 \mathrm{MPa}$ and $25 \mathrm{GPa}$, respectively, which is among the best mechanical performances reported for randomly-oriented CNT mats. We also develop a model accounting for radiation-induced hierarchical reinforcement of DWCNT films. The model is in good agreement with the experimental results across the entire dose range.
\end{abstract}

\footnotetext{
* Corresponding author. Tel: 925 422-0089. Email: fornasiero1 @1lnl.gov (Francesco Fornasiero) 


\section{Introduction}

Many natural materials feature remarkable structural properties, often exhibiting extraordinary toughness, strength, and stiffness in a light-weight composite. These superior mechanical properties are frequently realized through a complex hierarchical organization of relatively weak constituents [1-7]. In addition, functional diversity is achieved by geometrical variation of the assembly of a few building blocks, rather than relying on a large collection of different structural units $[3,6]$. A remarkable example is collagen, a stiff and hard protein that represents the most abundant constituent material in the human body. At the lower hierarchical level, collagen consists of polypeptide molecules arranged in a 300-nm long, $\sim 1.5$-nm wide triple-helix. At the next level, collagen molecules are quasi-hexagonally packed into $~ 50$-nm diameter interdigitated microfibrils, which then assemble to form millimeter long fibers with an $\sim 1-\mu \mathrm{m}$ diameter. Strength and stiffness of collagen provide mechanical stability to diverse tissues such as blood vessels, bones, cornea, skin, ligaments, and tendons as well as the extracellular matrix. The large diversity of mechanical properties of collagen connective tissues is dictated, to a large extent, by the spatial organization of the collagen fibers in 1-D (tendons and ligaments) or 2-D and 3-D network structures (skin and blood vessels) [7]. In addition, inter- and intra-fibril crosslinks $[1,3,8]$ enable efficient transmission of mechanical forces across multiple length-scales. Biomineral phases and/or other biopolymers further modulate the composite mechanical properties to optimally respond to primary tissue mechanical stresses.

The superior mechanical properties of natural materials have motivated research toward the development of artificial materials with biomimetic hierarchical design. Because of their excellent strength $(S)$ and elastic modulus $(E)$ approaching $\sim 100 \mathrm{GPa}$ and $\sim 1 \mathrm{TPa}$, respectively [9-12], and outstanding optical, thermal [13, 14], and electrical [15] properties, carbon nanotubes (CNTs) are very attractive 1-D nanoscale building blocks for designing lightweight, bio-inspired, multiscale-structured composites that could potentially outperform 
naturally occurring materials. In analogy with collagen, CNTs can be organized at the macroscale in 1-D fibers/yarns [16-18] or 2-D [19-21] and 3-D structures [22-26]. Moreover, CNTs assemble spontaneously in hexagonally packed bundles [21] that are reminiscent of collagen fibrils.

In spite of considerable recent progress in exploiting Nature lessons, macroscopic CNT composites still fail to fully deliver their promises, falling short a few orders of magnitude in their mechanical performance when compared with the pristine nanoscale building blocks [27, 28]. Strength, stiffness, and toughness of CNT-based macroscopic materials are limited by the weak interfacial interactions between CNT-based components, rather than by the properties of individual CNTs [29-31]. This deficiency is much more pronounced in 2-D and 3-D composites where requirements of isotropic mechanical, thermal, or electrical properties dictate the choice of a random CNT orientation [27, 32]. On the contrary, in CNT fibers [16, 33, 34] and oriented/pre-stretched CNT films [35-39], distribution of external load among CNTs is more efficient, often by nearly an order of magnitude. Thus, a critical challenge in assembling CNTs into high performance hierarchical structures is engineering the interfacial interaction across multiple length scales to favor load transfer between the nanosized constituents of the macroscale composite. Overcoming this challenge is expected to have a profound impact in aerospace and automotive applications, armor design, advanced thermal management composites, etc.

A promising approach to control interfacial interaction in CNT-based materials is to use light or particle irradiation for introducing strong covalent bonds among CNTs at multiple hierarchical levels as well as between CNTs and the hosting matrix [40-42]. For example, electron beam irradiation has been successfully employed to dramatically enhance the mechanical performance of individual multi-walled CNTs (MWCNT) by the formation of inter-shell crosslinks [11] and of bundles of single-walled CNTs (SWCNTs) and doublewalled CNTs (DWCNTs) via intertube links [43, 44]. UV irradiation was also demonstrated 
to be effective in increasing both electrical conductivity and shear modulus of SWCNT ropes by intertube cross-links without damaging the CNT graphitic structure [45]. These results suggest that similar strategies could be employed to promote efficient cross-linking of the building blocks at different hierarchical levels [46].

Motivated by such previous achievements [11, 43, 44], we investigate here the effect of high-energy $(3 \mathrm{MeV}) \mathrm{He}^{+}$ion irradiation on submicron-thick, large-area $\left(\sim 1 \mathrm{~cm}^{2}\right)$ DWCNT mats and DWCNT-Epoxy composites. In particular, rather than aiming at the optimization of the composite mechanical properties, we design our experiments toward elucidating the mechanism of irradiation-induced reinforcement in ultrathin films with randomly-oriented CNTs, i.e., for the most challenging structural organization of CNTs. In this study, the thickness of the DWCNT films and composites $(<0.5 \mu \mathrm{m})$ is chosen much smaller than the projected range of $3 \mathrm{MeV} \mathrm{He}^{+}$ions $(\sim 31$ and $15 \mu \mathrm{m}$ for neat DWCNT mats and DWCNTEpoxy composites, respectively, as calculated with the TRIM code [47]). This ensures that $\mathrm{He}^{+}$ions pass through rather than get implanted in the films and that radiation defects are generated uniformly through the film thickness. Moreover, such high-energy light ions create very dilute collision cascades and do not lead to irradiation-induced densification of CNTbased scaffolds, as in the case of heavy ions that generate dense collision cascades with much larger electronic energy loss [48].

By spanning three orders of magnitude in ion irradiation dose, we demonstrate that radiation-induced mechanical reinforcement can be associated with the formation of crosslinks at different hierarchical levels. The rapidly increasing reinforcement at low ion doses is attributed to intra-bundle CNT cross-links, while the following slow mechanical reinforcement at higher doses is associated with inter-bundle cross-links. At the highest irradiation doses of this study, DWCNT-Epoxy strength and elastic modulus approach 900 $\mathrm{MPa}$ and $25 \mathrm{GPa}$, respectively, which are among the best performances reported for composites based on randomly-oriented CNT mats [35, 49, 50]. Even higher stiffness and 
strength could be potentially achieved in hierarchically-organized CNT films and composites by optimizing ion irradiation conditions. Finally, we develop a mathematical model accounting for the fundamental physics of radiation-induced mechanical reinforcement. Our model is in good agreement with the experimental results across the entire ion dose range of this study.

\section{Experimental Section}

\subsection{Pure CNT films and CNT/epoxy composites}

The randomly-oriented CNT networks were produced with a floating catalyst CVD method. A toluene solution (carbon source) containing ferrocene (catalyst) and thiophone (cocatalyst) was vaporized with an atomizer and introduced into a custom-made horizontal ceramic-tube furnace with a controlled hydrogen stream $(2 \mathrm{sccm})$. Argon was used as inert carrier gas at a flow rate of $10 \mathrm{sccm}$ (Fig. 1A). The CNT growth temperature was $1100 \mathrm{C}$. Hundreds-of- $\mu \mathrm{m}$-long CNTs were collected on a nitrocellulose filter membrane at the end of the floating catalyst CVD system by applying slight vacuum. Produced CNTs are doublewalled with an outer diameter of 2-3 $\mathrm{nm}$ as evidenced by high resolution TEM imaging and supported by the location of clear radial breathing modes in the range $\omega=110-250 \mathrm{~cm}^{-1}$ of the Raman spectrum. According to the relation $\omega\left[\mathrm{cm}^{-1}\right]=224 / \mathrm{d}+14[\mathrm{~nm}][51]$, these frequencies correspond to $0.95-2.3 \mathrm{~nm}$ inner and outer tube diameters.

Free-standing epoxy/CNT composites were prepared by first transferring the as-deposited CNT networks onto a $125-\mu \mathrm{m}$ thick polyimide frame $(1.27-\mathrm{cm} \times 1.90-\mathrm{cm})$ with a $0.7-\mathrm{cm} \times 1.0-$ $\mathrm{cm}$ window that hosts the resulting suspended film. The transfer onto the polyimide frame was facilitated by spin-coating epoxy (EpoThin ${ }^{\mathrm{TM}}$ - Buehler) on the edge of the frame (3000 $\mathrm{rpm} / 1 \mathrm{~min}$ ) and by pre-curing the epoxy for 3-h at room temperature to reach the desired tackiness. After fully curing the adhesive, $20 \mu \mathrm{l}$ of a freshly prepared epoxy-ethanol solution 
(1 w\% of EpoThin ${ }^{\mathrm{TM}}$ resin+hardener in a 5:2 ratio) were drop cast onto the entire CNT film surface and let dry/cure overnight.

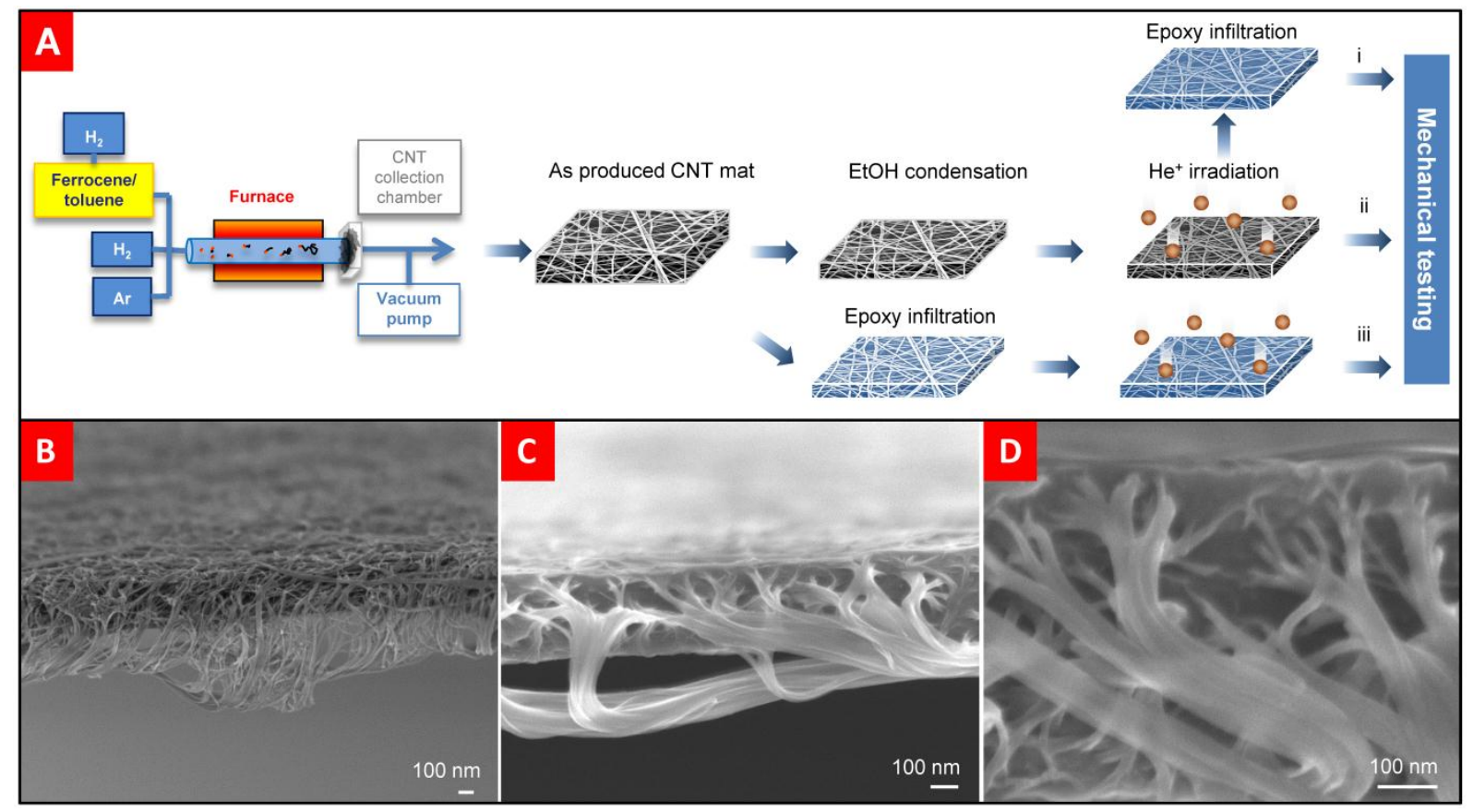

Fig. 1. Fabrication of DWCNT mats and DWCNT-epoxy composites: (a) Schematic representation of the production of DWCNT mats with a floating catalyst method, followed by ethanol densification or epoxy infiltration. Resulting thin films are exposed to $3 \mathrm{MeV} \mathrm{He}^{+}$ion irradiation before or after epoxy infiltration. Ethanol-densified DWCNT networks (ii $=$ DWCNT-EtOH), irradiated epoxy composites (iii $=$ DWCNT-Epoxy) and composites formed by epoxy infiltration after DWCNT network irradiation ( $\mathrm{i}=$ DWCNTirr-Epoxy) are then subjected to uniaxial tensile tests. (b-d) SEM cross-sectional images of a DWCNT-EtOH film (b) and DWCNTEpoxy composites (c-d). High resolution image in (d) clearly shows DWCNT bundles emerging from the composite cross-sectional surface.

Pure CNT films (DWCNT-EtOH) for mechanical testing were prepared by first wetting the CNT film with ethanol, drying the film overnight, and then applying the same transfer procedure to a polyimide frame as described above. The ethanol treatment densifies the otherwise weak CNT film and enables easy-handling. A similar film compaction occurs during the preparation of the DWCNT-Epoxy composites when the 1:100 epoxy-ethanol solution is allowed to dry at room temperature. Free-standing composite films of irradiated DWCNTs (DWCNTirr-Epoxy) were produced by first preparing free-standing, ethanol- 
treated CNT-films, exposing them to ion irradiation, followed by the infiltration with $20 \mu \mathrm{l}$ of the 1:100 epoxy-ethanol solution.

CNT loading in the epoxy composites was measured with a combination of Rutherford backscattering spectrometry (RBS) and elastic recoil detection analysis (ERDA). With RBS and ERDA, we quantify the total mass of carbon, hydrogen, nitrogen, oxygen, and iron catalyst in the CNT networks before and after epoxy infiltration. Because Fe is originally present only in the DWCNT films, the ratio (wt\% Fe in the composite) / (wt\% Fe in the DWCNT film) gives the weight fraction of DWCNTs in the composite.

\subsection{Raman Spectroscopy and Electron Microscopy}

Raman spectra were collected with a Nicolet Almega XR Dispersive Raman Spectrometer (Thermo Scientific) at a 632.8-nm HeNe excitation laser (1.96 eV). Every Raman measurement was conducted at room temperature, and the laser power levels were kept at $1 \%(0.1 \mathrm{~mW})$ to avoid excessive heating that could lead to CNT damage. A $100 \times$ objective lens was used to focus the laser beam on a $0.6-\mu \mathrm{m}$ spot of the CNT mat for an $80-\mathrm{s}$ total acquisition time. Raman spectra were collected at several different locations of the CNT and CNT/composite films. Reported D/G intensity ratios are averages of 3-6 spectra. Transmission electron microscopy (TEM) images were obtained with a FEI TF-20 Tecnai microscope, operated at $200 \mathrm{kV}$ with a $4.0 \mathrm{keV}$ extraction voltage for the field-emission gun. To prepare the samples for TEM imaging, CNTs were dry-deposited on a TEM grid by pressing/sliding the grid on the CNT film. Scanning electron microscopy images of CNT films and composites were collected at 5 or $10 \mathrm{kV}$ accelerating voltage with a JEOL 7401-F SEM. For cross-sectional imaging, samples were cracked in liquid nitrogen.

\subsection{Ion irradiation and ion beam analysis}

The $4 \mathrm{MV}$ ion accelerator (NEC, model 4UH) at Lawrence Livermore National Laboratory was used for both ion irradiation and ion beam analysis. All ion beam experiments 
were performed at room temperature. Irradiation was done with $3 \mathrm{MeV}{ }^{4} \mathrm{He}^{+}$ions to doses in the range of $10^{13}-10^{16} \mathrm{~cm}^{-2}$ with a constant beam flux of $10^{12} \mathrm{~cm}^{-2} \mathrm{~s}^{-1}$ in a surface normal direction. The elemental composition of films was studied by a combination of Rutherford backscattering spectrometry (RBS) and elastic recoil detection analysis (ERDA) [52]. Depth profiles of $\mathrm{Fe}, \mathrm{O}$, and $\mathrm{C}$ were measured by $\mathrm{RBS}$ with $2.0 \mathrm{MeV}{ }^{4} \mathrm{He}^{+}$ions incident normal to the sample surface and backscattered into a detector at $164^{\circ}$ relative to the incident beam direction. For hydrogen depth profiling, ERDA with a 3.0 $\mathrm{MeV}^{4} \mathrm{He}^{+}$ion beam was used. The sample normal direction was tilted to $70^{\circ}$ with respect to the incident beam direction, and hydrogen atoms recoiled at $150^{\circ}$ were measured with a surface barrier detector covered with a $10-\mu \mathrm{m}$-thick carbon foil. Analysis of RBS and ERDA spectra was done with stopping powers and scattering cross sections from the RUMP code [53] and from Reference [54], respectively. Ion ranges were calculated with the TRIM code (version SRIM-2011.08) [55].

\subsection{Mechanical testing}

Tensile samples were fabricated by mounting DWCNT films and composites on polyimide frames, as described above. The frames have holes at top and bottom that match pins in the test grips and serve as the datum for cutting the gage section of the sample. The sample profile was cut by laser ablation; both excimer and femtosecond lasers were used successfully. In order to prevent damage to the samples, the sides of the frames were cut away with sharp scissors after mounting in the grips. The samples were tested in an electromechanical load frame at a nominal strain rate of $10^{-3} \mathrm{~s}^{-1}$. A $2 \mathrm{~N}$ load cell was used to measure the force. The approximate sample strain was calculated from the compliance-corrected machine deflection. 


\section{Results and Discussion}

\subsection{Morphology of as-grown DWCNT films and their composites}

Randomly-oriented CNT networks were produced with a floating catalyst method in a custom-made chemical vapor deposition (CVD) reactor with ferrocene as the catalyst precursor and toluene as the hydrocarbon feedstock. The nanotube network consists of $\sim 0.1$ $1.0 \mathrm{~mm}$ long, 2-3 nm wide DWCNTs of very high crystalline quality, as evidenced by transmission electron microscopy (TEM) and Raman spectroscopy characterization (Fig. 2). The intensity ratio of the $\mathrm{G}$ and $\mathrm{D}$ bands before irradiation, $\mathrm{I}_{\mathrm{G}} / \mathrm{I}_{\mathrm{D}}(\mathrm{Fig} .2 \mathrm{~F})$, is in the range of 35-60. The as-produced DWCNT network contains $\sim 0.8-2.0$ at.\% of Fe catalyst particles (Fig. 2D), as measured by Rutherford backscattering spectrometry (RBS).

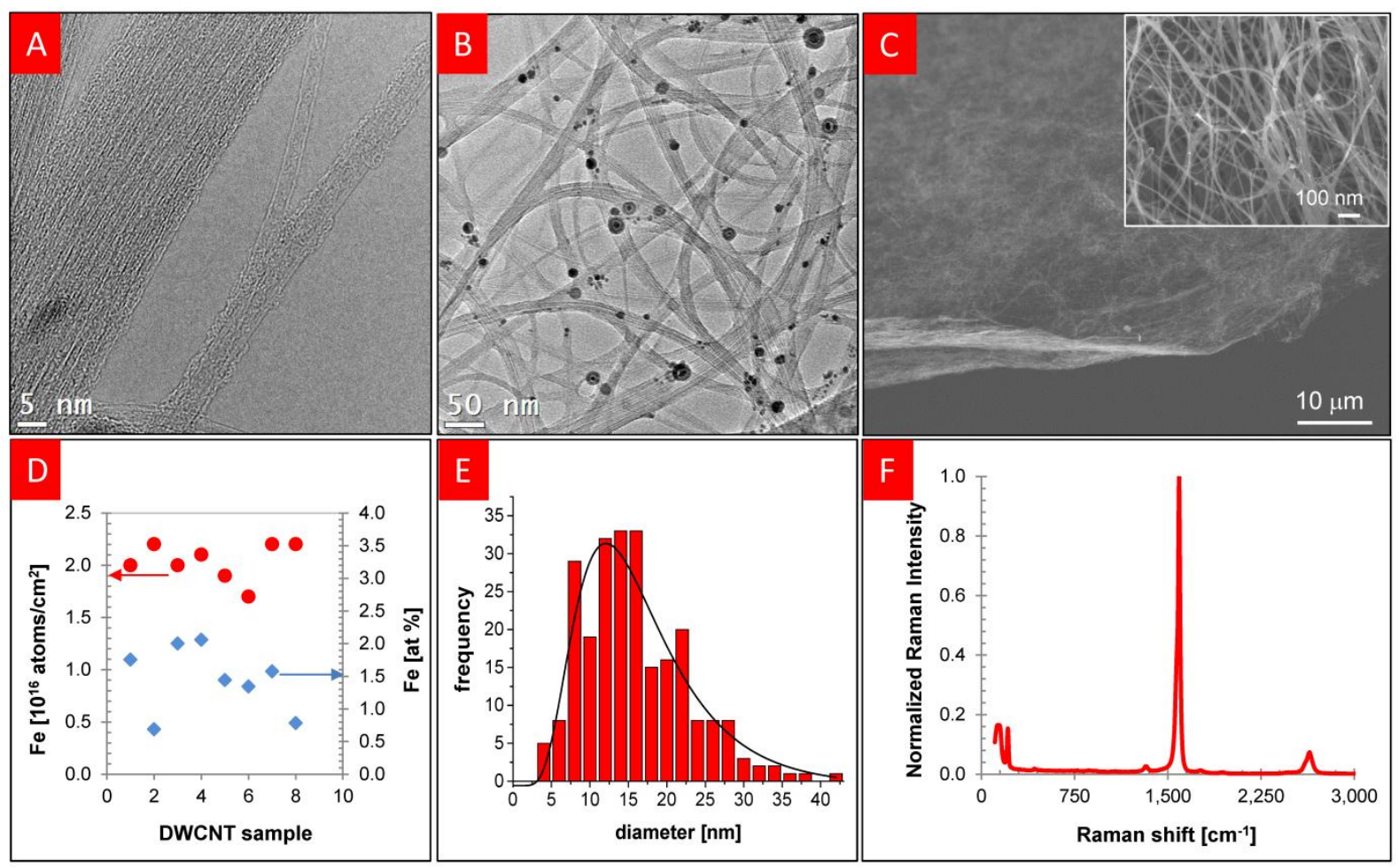

Fig. 2. Characterization of the hierarchically organized DWCNT mats: (A) HRTEM image of an 2-nm wide DWCNT merging into a bundle; (B) representative TEM image used to determine the DWCNT bundle diameter distribution in (E) and showing an entangled network of DWCNTs decorated with Fe catalyst particles; (C) low (main) and high (inset) magnification SEM images of the DWCNT network; (D) Fe content of eight different DWCNT films measured by RBS; (E) the distribution of bundle diameters determined by TEM imaging of $\sim 250$ bundles; (F) Raman spectrum of the DWCNT network, measured with a 632.8-nm laser excitation showing a high graphitization level and the presence of well-defined radial breathing modes in the range of $110-250 \mathrm{~cm}^{-1}$. 
Fig. 2A-C shows the hierarchical organization of the CNTs in the network. Small-radius DWCNTs form bundles with a lognormal diameter distribution in the range of 5-30-nm and centered on $\sim 15-\mathrm{nm}$, as determined by TEM imaging. In some cases, smaller bundles merge into larger bundles and share nanotubes. During the floating catalyst growth (Fig. 1A) [20, 56], long CNT bundles are deposited on the collecting filter paper with no preferential orientation, thus forming a continuous, randomly entangled network. This reticulate network assembly of long, highly crystalline DWCNTs is particularly favorable for the fabrication of high strength and modulus composite materials $[20,35,36,50,56]$. Because as-grown DWCNTs are essentially defect-free, the individual tubes are expected to have Young's modulus and strength approaching their theoretical maxima of $\sim 1 \mathrm{TPa}$ and $\sim 100 \mathrm{GPa}$, respectively [9-12]. When compared with CNT-reinforced composites fabricated by mixing polymers with short tubes, the reticulate structure has an advantage of promoting an even distribution of applied loads over a macroscopic area by continuously transferring stresses through the network rather than merely through weak polymer/CNT interfaces $[20,36]$.

As-grown DWCNT mats are highly porous, as evidenced in Fig. 2C, and thus very mechanically compliant and fragile. To increase the mat density for facile handling, as-grown DWCNT mats were first subjected to ethanol $(\mathrm{EtOH})$ condensation to form films with thickness in the range of $200-400 \mathrm{~nm}$. We will refer to them as DWCNT-EtOH films. Thin films of CNT-epoxy composites ( 300-nm-thick) were fabricated with an analogous procedure by simultaneously densifying and infiltrating the DWCNT network with a highly diluted epoxy-EtOH solution. Fig. 1C-D shows typical scanning electron microscopy (SEM) cross-sectional images of a fracture surface of a DWCNT-Epoxy composite film, clearly illustrating DWCNT bundles emerging from the fracture surface. The employed infiltration protocol resulted in composites with $45 \sim 60 \mathrm{wt} \%$ DWCNT, as measured by compositional analysis with a combination of RBS and elastic recoil detection analysis (ERDA). 


\subsection{Mechanical properties of un-irradiated DWCNT films and composites}

Elastic moduli and strength (defined as the stress at failure) for all samples (irradiated and not) were calculated on the basis of uniaxial tensile tests and the physical thickness of films. For each sample, the latter was determined by cross-sectional SEM imaging as an average of 12-25 measurements taken at different locations along the film edge. Fig. 3 shows the strength and Young's modulus of the un-irradiated epoxy films, DWCNT-EtOH films, and DWCNTEpoxy composites.

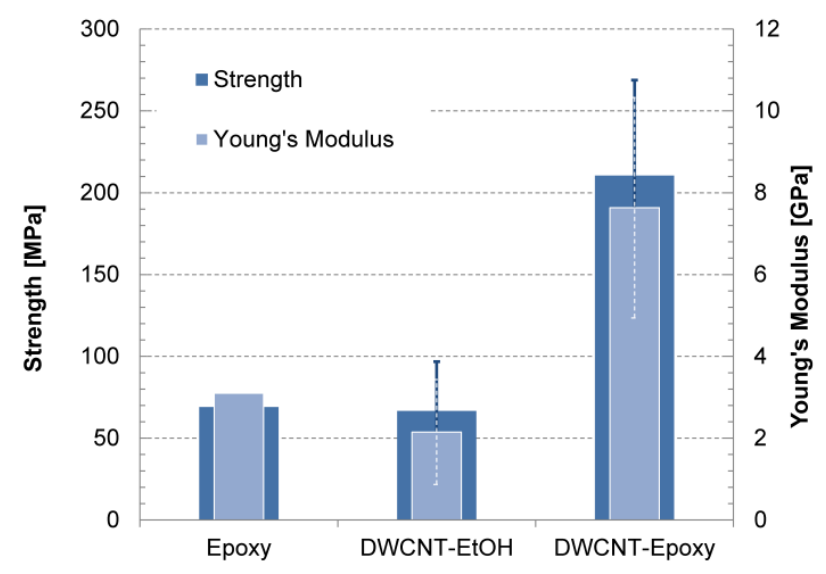

Fig. 3. Tensile strength and elastic modulus of the neat DWCNT network, epoxy films, and their composites before irradiation.

Fig. 3 reveals that the composite material has mechanical properties that greatly surpass those of its building blocks: the elastic modulus and strength of the composite films average $~ 7.6$ $\mathrm{GPa}$ and $210 \mathrm{MPa}$, respectively, while the corresponding two metrics are $E \sim 3 \mathrm{GPa}$ and $S \sim 70$ MPa for epoxy films, and $E \sim 2 \mathrm{GPa}$ and $S \sim 67 \mathrm{MPa}$ for DWCNT-EtOH films. Our result is consistent with recent reports for polymer composites with high loading of CNTs with reticulated architecture [36] and can be interpreted with the Halpin-Tsai model [57] with the assumption that the reinforcing fibers are nanotube bundles with an average diameter of 15 $\mathrm{nm}$ and length $>100 \mu \mathrm{m}$. A fit to the mechanical properties measured for DWCNT-Epoxy 
(with an $\sim 50 \%$ tube volume fraction) gives a reasonable elastic modulus ( 28 GPa) of the DWCNT bundles [44].

Nevertheless, both DWCNT-EtOH films and DWCNT-Epoxy composites have mechanical properties that are significantly inferior to those expected based on the properties of individual CNTs. Indeed, the Halpin-Tsai model predicts a Young's modulus of $190 \mathrm{GPa}$ for DWCNT-Epoxy composites having 2-nm-wide DWCNTs $(E=1 \mathrm{TPa})$ as reinforcing fibers. Similarly, the density scaling model for porous solids predicts a modulus of >100 GPa for our DWCNT-EtOH films [58]. Thus, the macroscopic mechanical response of DWCNT films and their composites remains largely dominated by weak interactions between shells in a nanotube, nanotubes in a bundle, bundles in the network, and of nanotubes with the matrix [29-31]. Weak coupling between the epoxy matrix and the DWCNTs is supported by crosssectional SEM images that reveal a pull-out of long CNT bundles from the fracture surface (Fig. 1C and S1A).

\subsection{Irradiation-induced reinforcement of DWCNT network and composites}

Efficient load transfer through the network of a DWCNT mat requires strong interactions between adjacent DWCNTs and CNT shells within DWCNTs. Electron beam irradiation at moderately low doses has previously been demonstrated to introduce stable links among CNTs in a bundle and among shells in MWCNTs, thus enabling impressive enhancements in the stiffness and strength of tubes and their bundles [11, 43, 44]. To promote covalent crosslinks not only at the tube and bundle level, but also among bundles in the porous network, we employed here high-energy $\mathrm{He}^{+}$ion irradiation $(3 \mathrm{MeV})$ in a wide dose range of $10^{13}-10^{16}$ ions $/ \mathrm{cm}^{2}$. Films of pure epoxy, ethanol-densified DWCNTs, and DWCNT-Epoxy composites were exposed to ion irradiation, and their mechanical properties were evaluated by uniaxial tensile tests. Also, to decouple the effect of ion irradiation on the strength of the interfacial interaction between CNTs and the epoxy matrix in composites, for selected samples, we first 
irradiated the CNT network and then infiltrated the network with epoxy. Such samples will be referred to as DWCNTirr-Epoxy (Fig. 1A).

Fig. 4 summarizes Young's modulus and strength for the four types of films as a function of ion dose, while corresponding tensile stress-strain plots are given in Fig. S2. It is seen from Fig. 4A that, for pure epoxy films $\left(\sim 1-\mu \mathrm{m}\right.$ thick), ion irradiation up to a dose of $2 \times 10^{15}$ $\mathrm{He}^{+} / \mathrm{cm}^{2}$ did not affect significantly the elastic modulus, while the epoxy strength steadily decreased with increasing ion dose (Fig. 4B). Epoxy films exposed to ion irradiation doses above $2 \times 10^{15} \mathrm{He}^{+} / \mathrm{cm}^{2}$ were too brittle for our tensile testing. These observations are consistent with previous studies of radiation effects in epoxy resins $[59,60]$.

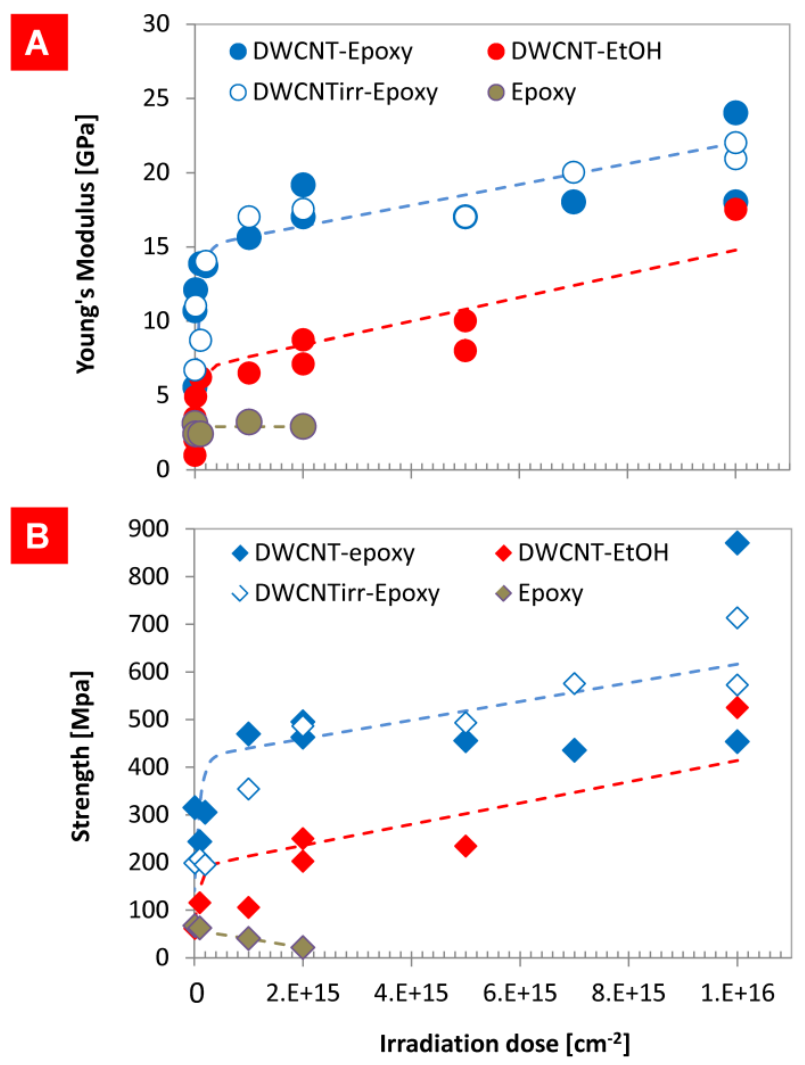

Fig. 4. Effect of $\mathrm{He}^{+}$ion irradiation dose on the mechanical properties of DWCNT-EtOH, DWCNTirr-Epoxy, DWCNT-Epoxy, and epoxy films. While elastic modulus (a) and tensile strength (b) for pure epoxy remain unchanged or decline with irradiation dose, DWCNT-containing films show a two-regime reinforcement process in which, at a rapid mechanical property increase at low doses follows a slow but steady strengthening and stiffening at high doses. Dashed lines are to guide the reader's eye. 
The radiation response of the samples containing CNTs is drastically different from that of pure epoxy films. For DWCNT-EtOH films, the elastic modulus increases very rapidly from $\sim 2$ to $\sim 6 \mathrm{GPa}$ at low doses (below $\sim 2 \times 10^{14}$ ions $/ \mathrm{cm}^{2}$ ). For higher doses, the modulus increases at a much slower rate, and there is no downturn of the mechanical performance of CNTcontaining films up to the largest dose of this study, $10^{16}$ ions $/ \mathrm{cm}^{2}$, at which $E$ reaches 17.5 GPa. This suggests that the DWCNT crystalline structure is conserved in the entire irradiation dose range. In agreement, the intensity ratio of the graphitic and defect Raman bands of our DWCNT network, $\mathrm{I}_{\mathrm{G}} / \mathrm{I}_{\mathrm{D}}$, remains $\geq 2$ for all the $\mathrm{He}^{+}$doses, and clear radial breathing modes are detectable in the Raman spectra even after $10^{16}$ ions $/ \mathrm{cm}^{2}$ (Fig. 5A). This is not unexpected. Indeed, due to a high ion energy and low ion mass, the position of the end-of-range damage peak of $3 \mathrm{MeV} \mathrm{He}$ ions is much deeper than the film thickness [47]. As a result, even the largest dose of this study $\left(10^{16}\right.$ ions $\left./ \mathrm{cm}^{2}\right)$ would generate only $\sim 0.002$ displacements per atom in the graphitic carbon matrix.

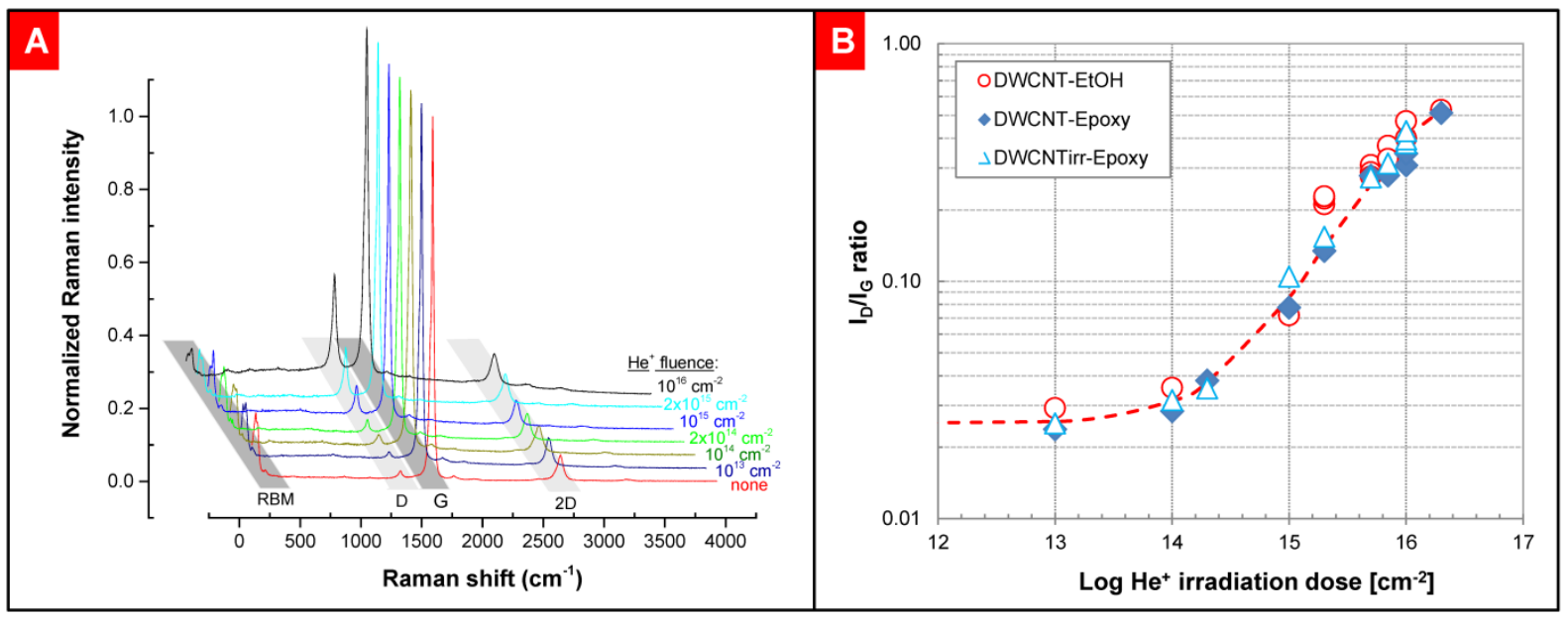

Fig. 5. Raman characterization of DWCNT-containing films as a function of ion irradiation: (a) Normalized Raman spectra for irradiated DWCNT-Epoxy films (G-band maximum intensity set =1). Similar results were obtained for DWCNT-EtOH and DWCNTirr-Epoxy films. (b) Irradiation dose dependence of the Raman bands intensity ratio, $\mathrm{I}_{\mathrm{D}} / \mathrm{I}_{\mathrm{G}}$, for DWCNT-EtOH, DWCNT-Epoxy, and DWCNTirr-Epoxy films. Nearly identical irradiation damage $\left(\mathrm{I}_{\mathrm{D}} / \mathrm{I}_{\mathrm{G}}\right)$ is observed for the three materials. The dashed line in $(\mathrm{b})$ is calculated with the defect overlap model in its simplest form of zero-overlap (Eq. (6) of Reference $\left.{ }^{[70]}\right): I_{D} / I_{G}=a+b\left(1-e^{-c * \Phi}\right.$ ), where $a, b$, and $c$ are constants and $\Phi$ is the irradiation dose. 
From the Raman intensity ratio $\left(\mathrm{I}_{\mathrm{D}} / \mathrm{I}_{\mathrm{G}}\right)$ of Fig. $5 \mathrm{~A}$, we can estimate the characteristic distance between defects in a CNT, $L_{D}[\mathrm{~nm}]$, and the areal density of defects, $\rho_{D}\left[\mathrm{~nm}^{-2}\right]$, with the following relations [61-64]:

$$
\begin{aligned}
& L_{D}^{2}=\left(1.8 \times 10^{-9}\right) \times \lambda_{\text {laser }}^{4} \times\left(\frac{I_{D}}{I_{G}}\right)^{-1}, \\
& \rho_{D}=1 /\left(\pi L_{D}^{2}\right),
\end{aligned}
$$

where $\lambda_{\text {laser }}$ is the Raman laser wavelength $(632.8 \mathrm{~nm})$. The smallest calculated $L_{D}$ is $\sim 25-\mathrm{nm}$, thus confirming that the graphitic order is maintained even in the most heavily damaged films. Because we did not see a downturn of the film mechanical performance up to $10^{16}$ ions $/ \mathrm{cm}^{2}$, further mechanical reinforcement is likely achievable at higher ion doses. Unfortunately, our attempts to verify this claim have not been successful because 300 -nm thick films exposed to $>10^{16} \mathrm{He}^{+} / \mathrm{cm}^{2}$ doses were too brittle for handling during tensile testing experiments due to the reduction in the maximum strain at failure with increasing ion dose (see Figs. S2 and S6).

A previous study of single MWCNTs exposed to an electron-beam has revealed that irradiation-induced changes to the elastic properties strongly depend on the quality of the tubes before irradiation [65]. For highly defective tubes, the bending modulus decreased steadily with irradiation dose, whereas, for MWCNTs of intermediate quality, the bending modulus first increased before dropping at large irradiation doses. For the highest quality, smallest diameter MWCNTs, no downturn of the mechanical properties was observed within the irradiation dose range of that study $\left(<1 \times 10^{20} \mathrm{e} / \mathrm{cm}^{2}\right)$. Similarly, the bending modulus of SWCNT bundles displayed a maximum when exposed to doses up to $2 \times 10^{22} \mathrm{e} / \mathrm{cm}^{2}$ [43]. These trends were explained with the following argument: when the overall damage on the tube walls is too extensive, the cross-links formed during electron-beam irradiation can connect only short segments of the graphitic shells and cannot form a percolation pathway of covalent bonds from one CNT end to the other [65]. Thus, the bending modulus of irradiated MWCNTs declines above a threshold dose, the magnitude of which increases with the quality 
of the starting CNT tubes. These previous results are fully consistent with our findings. The small diameter DWCNTs of our work are highly crystalline and, even at the highest irradiation dose, the defect density estimated from Raman spectroscopy remains low and comparable with that of good quality MWCNTs produced by chemical vapor deposition, which typically have a $I_{D} / I_{G}<3[66,67]$. The onset of a downturn of the elastic properties of our DWCNTs films is expected to require $\mathrm{He}^{+}$ion doses larger than those used here.

The shape of the dose dependence of the DWCNT-EtOH strength, shown in Fig. 4B, mirrors that of the elastic modulus from Fig. 4A. However, strength data suffer from a larger experimental error, especially for the highest irradiation dose used, where early film failure was often observed. At the highest irradiation dose of this study, the strength of irradiated DWCNT-EtOH approaches $525 \mathrm{MPa}$.

Fig. 4 further reveals that, within experimental uncertainty, DWCNT-Epoxy (irradiation applied post epoxy infiltration) and DWCNTirr-Epoxy (irradiation applied to mats pre-epoxy infiltration) films have identical mechanical properties in the entire dose range. These results indicate that the irradiation-induced mechanical reinforcement in the composite material is almost exclusively due to crosslinks forming among the CNTs in the network, whereas the contribution from the possible irradiation-induced creation of CNT-matrix interfacial bonds is negligible. Moreover, cross-sectional SEM imaging (Fig. S1B) of DWCNT-Epoxy samples irradiated at the highest dose $\left(10^{16}\right.$ ions $\left./ \mathrm{cm}^{2}\right)$ shows DWCNT bundle pull-out from the fracture surface that closely resembles the behavior of un-irradiated DWCNT-Epoxy composites (Fig. S1A). In Fig. 4, mechanical properties of irradiated CNT composites follow the same trend as DWCNT-EtOH films. The maximum recorded Young's modulus and strength of the composite films are, however, much higher, reaching $24 \mathrm{GPa}$ and $870 \mathrm{MPa}$, respectively, for a dose of $10^{16}$ ions $/ \mathrm{cm}^{2}$. These match the best values reported so-far for macroscopic samples of isotropic, un-irradiated CNT-composites [35, 49, 50], surpass those of electron-beam-irradiated SWCNT films [68], and approach those of MWCNT yarns and 
SWCNT ropes exposed to gamma-ray irradiation (Fig. S3) [69] and to UV irradiation [45], respectively. Noteworthy, the reinforcement by $\mathrm{He}^{+}$ion bombardment described here has been achieved for ultrathin, isotropic DWCNT mats and composites without loss of the CNT graphitic structure and without any measureable film densification (Fig. S4), further pointing to the formation of cross-links as the primary mechanism of mechanical properties enhancement.

The Raman analysis performed for the DWCNT-EtOH samples was also done for DWCNT-Epoxy composites. Since epoxy is not Raman active in the Raman shift range of interest, the $\mathrm{I}_{\mathrm{D}} / \mathrm{I}_{\mathrm{G}}$ ratio did not change after adding epoxy to DWCNT-EtOH films (Fig. S5). Hence, Eq. (1) and (2) can be used to quantify the radiation-produced defect density in DWCNTs contained in DWCNT-Epoxy composites. Fig. 5B shows that $\mathrm{I}_{\mathrm{D}} / \mathrm{I}_{\mathrm{G}}$ ratios for all three CNT-containing films (i.e., DWCNT-EtOH, DWCNT-Epoxy, and DWCNTirr-Epoxy) follow the same master curve as a function of ion dose. The accumulation of defects with increasing irradiation dose is well described by the defect overlap model of cylindrical tracks (the dashed line in Fig. 5B) in its simplest case of so-called zero overlap (Eq. (6) of Reference [70], with a constant, non-zero defect density in our un-irradiated films).

Thus, these results suggest that a) high-energy $\mathrm{He}^{+}$ion irradiation is an effective strategy to dramatically enhance mechanical properties of thin films of randomly-oriented DWCNTs (8 fold increase) and DWCNT-epoxy composites (3 fold increase); b) the reinforcement of DWCNT containing films is due to the formation of CNT cross-links during ion bombardment; c) defects produced by different $\mathrm{He}^{+}$ions are not interacting, and d) DWCNTs that were irradiated while embedded in the polymer matrix maintain a high degree of crystallinity up to the maximum dose of this study, $10^{16}$ ions $/ \mathrm{cm}^{2}$.

Elongation at break $\left(\varepsilon_{b}\right)$ and toughness $(T)$ of irradiated epoxy, DWCNT films, and their composites were also monitored as a function of the irradiation dose (Fig. S6). Despite some scatter of the data, a few trends were noted. For epoxy films, the already modest $\varepsilon_{b}(\sim 3.5 \%)$ 
and $T\left(1.6 \mathrm{~J} / \mathrm{cm}^{3}\right)$ displayed a rapid and continuous decay by $1-2$ orders of magnitude at the highest dose $\left(2 \times 10^{15}\right.$ ions $\left./ \mathrm{cm}^{2}\right)$, whereas elongation at break and toughness of DWCNT containing films plateaued at $\varepsilon_{b} \sim 3 \%$ and $T \sim 10 \mathrm{~J} / \mathrm{cm}^{3}$, respectively, for doses up to $10^{16}$ ions $/ \mathrm{cm}^{2}$. In spite of the brittle epoxy matrix, composites with DWCNTs were tougher than the corresponding building blocks, and in several instances at low $\mathrm{He}^{+}$doses, the recorded toughness values $\left(30-117 \mathrm{~J} / \mathrm{cm}^{3}\right)$ approached those of the best randomly-oriented CNTpolymer films reported so far ([49] and references therein). Before irradiation and at small doses, the elongation at break for both DWCNT-EtOH and DWCNT-epoxy films was large and in excess of $45 \%$.

Thus, the addition of DWCNTs to epoxy dramatically enhances the material toughness, and strong composites are achieved at all irradiation conditions. However, at large irradiation doses, the material stiffening is compensated by a comparable decrease in the elongation at break, thus resulting in a toughness plateau.

\subsection{Modeling ion-irradiation reinforcement of DWCNT random networks}

The experimental data of Fig. 4 have revealed two regimes in the mechanical property enhancement of randomly-oriented CNT mats when exposed to $\mathrm{He}^{+}$ion irradiation over a dose range of three orders of magnitudes $\left(10^{13}-10^{16}\right.$ ions $\left./ \mathrm{cm}^{2}\right)$. In the first regime, for doses up to $\sim 2 \times 10^{14}$ ions $/ \mathrm{cm}^{2}$, the stiffness and strength rapidly increase with dose. In the second regime, corresponding to larger doses, the reinforcement proceeds at a much reduced, nearlyconstant rate. We attribute the origin of these two regimes to radiation-induced cross-linking of CNTs at different hierarchical levels of the mat. At the level of individual DWCNTs, the irradiation-induced formation of covalent bonds between the two concentric shells in a DWCNT effectively distributes stresses from the outer to the inner shell, which are otherwise weakly coupled by van der Waals interactions $[11,71,72]$. At the next hierarchical level (the bundle), irradiation results in cross-links among the DWCNTs of the bundle, dramatically 
increasing bundle shear strength and stiffness and preventing tube pull-out [29, 30, 43, 44, 73]. At the network level, inter-bundle covalent bonds formed by irradiation reinforce the CNT mat by strengthening the bundle-to-bundle contact points and limiting inter-bundle slippage [30]. Because shells within a DWCNT and DWCNTs within a bundle are closely packed for a large part of (if not for the entire) tube/bundle length, we expect that relatively low irradiation doses will be sufficient to generate a number of inter-shell and intra-bundle covalent bonds that maximize the reinforcement achievable at the tube/bundle level. Indeed, simulations of cross-linked DWCNTs suggest that a relatively small number of inter-shell covalent crosslinks can transfer load very efficiently between shells [11, 30]. On the opposite, the contact area between two bundles is much smaller, on the order of $\sim 1 \mathrm{~nm}^{2}$ (for bundles that are not sharing tubes). Thus, it is statistically less likely to link bundles together by irradiation, and saturation of the total strength of the inter-bundle contacts is expected to require much higher irradiation doses.

According to this picture, the regime of the steeply increasing elastic modulus and strength at low doses corresponds primarily to the formation of inter-shell and intra-bundle covalent bonds. Such reinforcement at tube and bundle levels saturates at $\sim 2 \times 10^{14} \mathrm{ions} / \mathrm{cm}^{2}$. A relatively small number of inter-bundle crosslinks are also created in this regime. At higher doses, the enhancement of the network mechanical properties originates primarily from interbundle crosslinks that are formed at a much slower rate.

The chemical nature and configuration of the bonds activated by $\mathrm{He}^{+}$ion irradiation are challenging to study experimentally. However, previous first principle simulations suggest that a variety of irradiation-induced defects could bridge the gap between neighboring CNTs in a bundle [43] and between shells in MWCNTs [11]. These defects include vacancies [74], interstitial carbon atoms [43, 74], Frenkel pairs or Wigner defects [73, 74], and native carboxyl groups [43], all of which may be formed in our irradiated mats. 
Based on the physical interpretation of the trends observed in our experiments, we construct an analytical model that accounts for the key features of our material and the irradiation process (Fig. 6). Following recent work of Åström et al. [75, 76], we model the DWCNT mat as a network of randomly oriented bundles of equal length, $L$, and width, $w$. The bundles are assumed to be rigidly bonded at the contact points where ion irradiation has formed a covalent crosslink. We divide each bundle into segments. Each segment represents a section of the bundle between two crosslinks. The length distribution of such segments is approximated by a 1-D Poisson distribution [76]. By calculating the total elastic energy of the mat as the sum of elastic energies of the segments under the assumption that the segments deform only via their least stiff mode, Åström et al. [75-77] derived the following expression for the Young's modulus of the mat, $E_{\text {mat }}$ :

$$
E_{\text {mat }}(z)=\frac{E_{\text {bundle }} w}{8 L} q\left\{\left(\frac{z w}{l_{c}}\right)^{2} E_{1}(z)+\left(3+\frac{1}{2(1+v)}\right)\left[1-e^{-z}(z+1)\right]\right\}
$$

where $E_{\text {bundle }}$ is the Young's modulus of the bundle; $q$ is the areal density of CNT bundles; $v$ is the mat's Poisson ratio; $l_{c}$ is the critical segment length above which the segments deform only by bending and below which only by stretching and shearing; and $z$ is a dimensionless parameter related to ion dose via the number of irradiation-induced crosslinks per nanotube, $n_{\text {links }}$, by $z=\frac{l_{c}}{L} n_{\text {links }}$. In Eq. (3), $E_{1}(z)$ is defined by $E_{1}(z)=\int_{1}^{\infty} e^{-z * x} / x d x$ and $l_{c}$ is calculated from $l_{c}=(1.9 * w+0.11) * \sqrt{2 *(1+v)}$, respectively $[75,76]$.

Eq. (3) accounts only for the irradiation-induced reinforcement associated with interbundle crosslinks. Since the network has a finite elastic modulus before irradiation, we assume that the un-irradiated mat has $n_{0}$ "effective" crosslinks that are related to the mat density by $n_{0}=2 q / \pi$. 


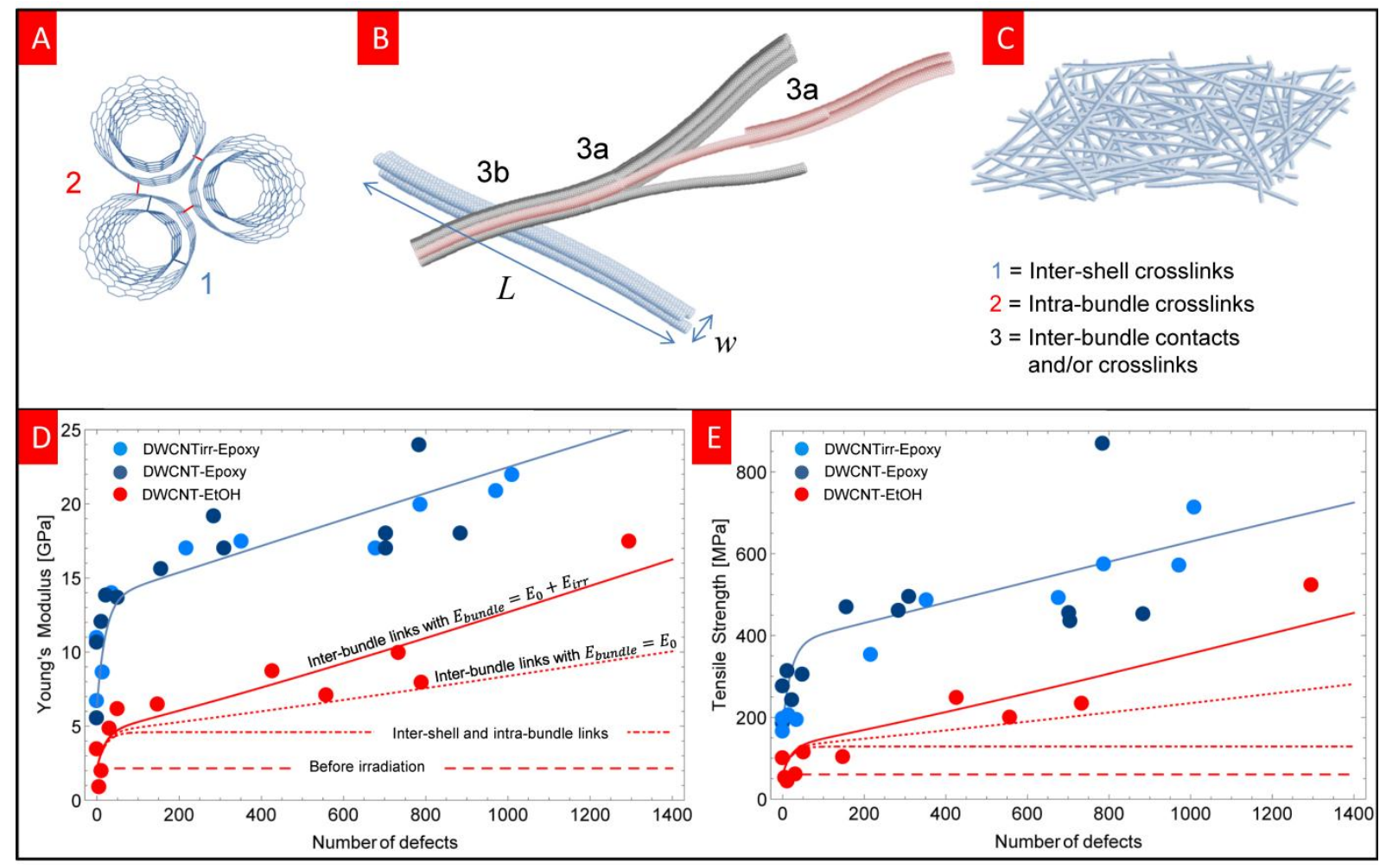

Fig. 6. Schematic representation of the hierarchical reinforcement mechanism of DWCNT-containing films upon irradiation and corresponding model calculations. (A) Reinforcement due to inter-shell (1, in blue) and intrabundle (2, in red) crosslinks are represented by Eq. (4). (B) Inter-bundle cross-links at bundle contact points are accounted for by Eq. (3). Bundles may (3a) share nanotubes and merge into larger bundles or (3b) simply contact at crossing points. (C) Model representation of the DWCNT network as an isotropic 2-D film made of rods (bundles) with length $L$ and width $w$. (D-E) Reinforcement of DWCNT-EtOH at different hierarchical level. Dashed lines: mechanical properties of the DWCNT-EtOH films before irradiation. Dot-dash lines: the contribution of irradiation-induced intra-bundle and inter-shell cross-links calculated with Eq. (3) and (4) by evaluating $E_{\text {mat }}$ at $Z_{0}$ (no inter-bundle links) and $E_{\text {bundle }}$ at $z$. Dotted lines: additional reinforcement due to interlinking bundles with Young's modulus equal to the un-irradiated value, $E_{\text {bundle }}=E_{0}$. If the bundle Young's modulus is set to its saturation value at high irradiation doses (i.e., $E_{\text {bundle }}=E_{0}+E_{\text {irr }}$ ), the same calculation yields DWCNT-EtOH mechanical properties that are indistinguishable from the full model results (solid lines). Solid lines: fitting of the experimental (D) Young's moduli and (E) tensile strengths with Eq. (1-5). The number of defects at different irradiation doses (x-axis) is calculated with Eq. (1-2) and (5) with a bundle length of $L=$ $400 \mu \mathrm{m}$.

Thus, in our model, we modify Eq. (3) by replacing $z$ with $\left(z+z_{0}\right)$, where $z_{0}=\frac{l_{c}}{L} n_{0}$. Furthermore, to describe the reinforcement at the tube/bundle level due to the formation of inter-shell and intra-bundle $s p^{3}$ bonds, we assume that $E_{\text {bundle }}$ increases with the number of crosslinks generated by ion irradiation according to 


$$
E_{\text {bundle }}(z)=E_{0}+E_{\text {irr }}\left(1-e^{-c * Z}\right)
$$

where $E_{0}$ is the bundle Young's modulus before irradiation; $E_{\text {irr }}$ represent the maximum enhancement of the elastic modulus of the bundle upon irradiation, and $c$ is constant that defines the rate of the bundle stiffness increase with dose. The mathematical form of Eq. (4) is justified by recent experimental measurements of low-dose electron irradiation effects on DWCNT bundles of sizes matching those of our DWCNT mat distribution: the Young's modulus and tensile strength display an "approximately exponential increase" with electron irradiation dose in the low-dose regime [44]. In addition, simulations of SWCNT bundles have revealed a similar trend for the bundle elastic modulus with the number of crosslinks [78]. While higher irradiation doses are expected to deteriorate the mechanical properties of CNTs by the degradation of the intrinsic crystalline structure of DWCNT shells (an effect not accounted for in Eq. (4)), Raman measurements reveal that our DWCNTs maintain their well graphitized walls even at the highest irradiation doses studied: the Raman G/D intensity ratio is $\geq 2$ even when the CNT mat is exposed to $10^{16}$ ions $/ \mathrm{cm}^{2}$ (Fig. 5).

For applying this model to fit our experimental data, we first need to estimate $n_{\text {links }}$, the number of crosslinks (i.e., covalent bonds) per nanotube introduced by $\mathrm{He}^{+}$ion irradiation. We assume here that $n_{\text {links }}=\alpha * n_{\text {defects }}$, where $\alpha$ is a proportionality constant and $n_{\text {defects }}$ is the number of irradiation-induced defects per nanotube. The latter is estimated with the following relation:

$$
n_{\text {defects }}=\rho_{D} * 2 \pi R_{C N T} L
$$

where $R_{C N T}$ is the DWCNT radius $(\sim 1 \mathrm{~nm})$ and $\rho_{D}$ is calculated with Eq. (1) and (2) from the measured Raman intensity ratio $\mathrm{I}_{\mathrm{D}} / \mathrm{I}_{\mathrm{G}}$ as a function of ion dose [61-63].

In our calculations, the number of crosslinks in un-irradiated samples obtained with Eq. (1-2) and (5) is subtracted out to compute the number of irradiation-produced defects. Also, 
we set the bundle length $(L=400 \mu \mathrm{m})$ and the bundle width $(w=15 \mathrm{~nm})$ to their average values measured by electron microscopy. For simplicity, we assume the Poisson ratio to be 0.33 . Results are not very sensitive to the choice of $v$ and $L$. To reduce the number of adjustable parameters down to $3\left(\mathrm{z}_{0}, \alpha, c\right)$, we use the measured un-irradiated DWCNT mat Young's modulus $E_{\text {mat }}(0)$ to express $E_{0}$ as a function of $z_{0}$ through Eq. (3). In addition, we impose the constraint that the fitted $\left(E_{0}+E_{\text {irr }}\right)$ must be equal to the maximum stiffening of DWCNT bundles under electron beam irradiation reported by Filleter and co-workers ( 693 GPa) [44].

Fitting our experimental data with Eq. (3) well describes the dose dependence of the Young's modulus (see Fig. 6A) of DWCNT-EtOH films. Fitting parameters and input constants are summarized in Table 1 . The calculated elastic modulus of un-irradiated bundles $\left(E_{0} \sim 324.3 \mathrm{GPa}\right)$ is $\sim 5-10$ times larger than quoted by Filleter et al.[44, 79]. This apparent discrepancy is attributed to the dramatically shorter length $(2-3 \mu \mathrm{m})$ of DWCNT bundles of References [44, 79] before irradiation [30, 44, 79]. Regardless, the agreement of the model with our experimental data in Fig. 6D is excellent.

Table 1. Model parameters and constants. ${ }^{\text {a) }}$

\begin{tabular}{ccc}
\hline Parameter/constant & DWCNT-EtOH & DWCNTirr-Epoxy \\
\hline$L[\mu \mathrm{m}]$ & 400 & 400 \\
$w[\mathrm{~nm}]$ & 15 & 15 \\
$z_{0}$ & $0.427^{\mathrm{b})}$ & $0.849^{\mathrm{b})}$ \\
$E_{0}[\mathrm{GPa}]$ & 324.3 & 324.3 \\
$E_{\text {irr }}[\mathrm{GPa}]$ & 368.7 & 368.7 \\
$c$ & $127.8^{\mathrm{b})}$ & 127.8 \\
$\alpha$ & $3.28^{\mathrm{b})}$ & 3.28 \\
\hline$\varepsilon$ & $0.028^{\mathrm{b})}$ & 0.028 \\
$\sigma_{0}=\varepsilon E_{0}[\mathrm{GPa}]$ & 9.08 & 9.08 \\
\hline
\end{tabular}

a) Eq. (1-5) are first applied to fit DWCNT-EtOH Young's modulus and extract the DWCNT bundle parameters $E_{0}\left(z_{0}\right), \alpha, c . \varepsilon$ is fitted to the irradiated, DWCNT-EtOH strength data with the Eq. $\sigma_{m a t}=\varepsilon \times E_{\text {mat }}$. For epoxy composites, $z_{0}$ is obtained by fitting the experimental elastic moduli in the entire range of irradiation dose; ${ }^{b}$ ) Quantities indicated with ${ }^{\text {b) }}$ are fitting parameters 
Fig. 6D also differentiates effects of introducing cross-links at different hierarchical levels on the total mechanical reinforcement during irradiation. Stiffening of the DWCNT bundles due to inter-shell and intra-bundle links rapidly increases the mat mechanical properties up to a saturation level. This contribution is calculated with Eq. (3) with a constant inter-bundle cross-link density $z=z_{0}$, and with Eq. (4) with $z$ increasing with ion dose. The stiffening rate due to the formation of inter-bundle cross-links depends on the mechanical properties of the bundles. If the DWCNT-bundle Young's modulus is set to its saturation value $E_{\text {bundle }}=$ $E_{0}+E_{i r r}$, the mat elastic moduli calculated with Eq. (3) match those obtained by fitting the full model to the experimental data (solid lines in Fig. 6D). Thus, these calculations confirm that the contribution of inter-bundle links to the films' mechanical reinforcement becomes dominant after the DWCNT bundle stiffness has already approached its maximum.

We have also estimated the mat strength with the relation $\sigma_{\text {mat }}=\varepsilon \times E_{\text {mat }}$, where $\varepsilon$ is a fitting constant. Fig. 6E shows that this relation well describes the strength data set within experimental error. As for the elastic modulus, the estimated maximum bundle strength ( 19.4 GPa) is very close to results of Filleter et al. [44], while the calculated bundle strength before irradiation (9.08 GPa) is 3-4 times higher than in References [44, 79].

As discussed in the previous section, the measured Young's modulus and tensile strength for irradiated composites of DWCNTs and epoxy suggest that a) the polymer matrix increases the overall mechanical properties by constraining the slip of CNTs and CNT bundles in the network, b) the irradiation-induced mechanical reinforcement in the composite material is due to crosslinks forming among the DWCNTs in the network and not to CNT-matrix interfacial bonds, and c) the number of CNT defects produced by ion irradiation in DWCNT-EtOH films and composites is nearly identical (see Fig. 5B and Eq. (2)). Thus, as shown in Fig. 6D-E, Eq. (3) can be used to quantitatively reproduce the experimental results for DWCNT-Epoxy films by adjusting only the parameter $z_{0}$ to account for DWCNT-EtOH mechanical properties increase upon epoxy infiltration. In summary, the proposed model appears to account for the 
essential physics dictating ion-irradiation reinforcement of randomly-oriented CNT networks and its composites, with model parameters and constants having clear physical meaning.

\section{Conclusion}

We have demonstrated a dramatic enhancement of mechanical properties of thin films of randomly-oriented DWCNTs and DWCNT-epoxy composites by irradiation with $3 \mathrm{MeV} \mathrm{He}^{+}$ ions. The irradiation-induced reinforcement behavior is characterized by two distinct regimes, which we have associated with the formation of cross-links at different DWCNT network hierarchical levels. Mechanical reinforcement is dominated by intra-bundle cross-links at low doses and by inter-bundle cross-links at high doses. At the highest irradiation dose of this study $\left(10^{16}\right.$ ions $\left./ \mathrm{cm}^{2}\right)$, the irradiated DWCNT-epoxy composites and neat DWCNT films have displayed a 3-fold and an 8-fold increase, respectively, in both tensile strength and elastic modulus with respect to values for the un-irradiated counterparts. At this ion fluence, the strength and elastic modulus of the composite (neat DWCNT film) approach 900 MPa and $25 \mathrm{GPa}(525 \mathrm{MPa}$ and $17.5 \mathrm{GPa})$, respectively, without loss of the DWCNT graphitic

structure. These mechanical performances are among the best reported for randomly-oriented CNT mats and could be further improved by optimizing ion irradiation conditions. Similar reinforcements are also expected during irradiation of materials containing aligned CNTs. Importantly, this mechanical reinforcement method relies on commercial ion implanters and, hence, is scalable to processing of large areas (300 mm wafers) and thicker films [80].

\section{Acknowledgements}

This work was supported by Lawrence Livermore National Laboratory. Lawrence Livermore National Laboratory is operated by Lawrence Livermore National Security, LLC, for the U.S. Department of Energy, National Nuclear Security Administration under Contract DE-AC5207NA27344. TEM experiments were conducted at the National Center for Electron 
Microscopy, Molecular Foundry, Lawrence Berkeley National Lab, which is supported by the Office of Science, Office of Basic Energy Sciences, of the U.S. Department of Energy under Contract \# DE-AC02-05CH11231.

\section{Supporting Information}

Supplementary data (Fig. S1-S5) associated with this article can be found in the online version. 


\section{References}

[1] Buehler MJ. Nature Designs Tough Collagen: Explaining the Nanostructure of Collagen Fibrils. Proc Natl Acad Sci U S A. 2006;103(33):12285-90.

[2] Espinosa HD, Rim JE, Barthelat F, Buehler MJ. Merger of Structure and Material in Nacre and Bone - Perspectives on de Novo Biomimetic Materials. Progress in Materials Science. 2009;54(8):1059-100.

[3] Gautieri A, Vesentini S, Redaelli A, Buehler MJ. Hierarchical Structure and Nanomechanics of Collagen Microfibrils from the Atomistic Scale Up. Nano Lett. 2011;11(2):757-66.

[4] Giesa T, Arslan M, Pugno NM, Buehler MJ. Nanoconfinement of Spider Silk Fibrils Begets Superior Strength, Extensibility, and Toughness. Nano Lett. 2011;11(11):5038-46.

[5] Keten S, Xu ZP, Ihle B, Buehler MJ. Nanoconfinement Controls Stiffness, Strength and Mechanical Toughness of Beta-Sheet Crystals in Silk. Nature Materials. 2010;9(4):35967.

[6] Nova A, Keten S, Pugno NM, Redaelli A, Buehler MJ. Molecular and Nanostructural Mechanisms of Deformation, Strength and Toughness of Spider Silk Fibrils. Nano Lett. 2010;10(7):2626-34.

[7] Meyers MA, Chen PY, Lin AYM, Seki Y. Biological Materials: Structure and Mechanical Properties. Progress in Materials Science. 2008;53(1):1-206.

[8] Orgel J, Irving TC, Miller A, Wess TJ. Microfibrillar Structure of Type I Collagen in Situ. Proc Natl Acad Sci U S A. 2006;103(24):9001-5.

[9] Yu MF, Lourie O, Dyer MJ, Moloni K, Kelly TF, Ruoff RS. Strength and Breaking Mechanism of Multiwalled Carbon Nanotubes Under Tensile Load. Science. 2000;287(5453):637-40.

[10] Yu MF, Files BS, Arepalli S, Ruoff RS. Tensile Loading of Ropes of Single Wall Carbon Nanotubes and Their Mechanical Properties. Phys Rev Lett. 2000;84(24):5552-5.

[11] Peng B, Locascio M, Zapol P, Li SY, Mielke SL, Schatz GC, et al. Measurements of Near-Ultimate Strength for Multiwalled Carbon Nanotubes and Irradiation-Induced Crosslinking Improvements. Nat Nanotechnol. 2008;3(10):626-31.

[12] Wei XL, Chen Q, Peng LM, Cui RL, Li Y. Tensile Loading of Double-Walled and Triple-Walled Carbon Nanotubes and their Mechanical Properties. Journal of Physical Chemistry C. 2009;113(39):17002-5.

[13] Kim P, Shi L, Majumdar A, McEuen PL. Thermal Transport Measurements of Individual Multiwalled Nanotubes. Phys Rev Lett. 2001;87(21):215502.

[14] Pop E, Mann D, Wang Q, Goodson KE, Dai HJ. Thermal Conductance of an Individual Single-Wall Carbon Nanotube Above Room Temperature. Nano Lett. 2006;6(1):96-100.

[15] Ebbesen TW, Lezec HJ, Hiura H, Bennett JW, Ghaemi HF, Thio T. Electrical Conductivity of Individual Carbon Nanotubes. Nature. 1996;382(6586):54-6.

[16] Behabtu N, Young CC, Tsentalovich DE, Kleinerman O, Wang X, Ma AWK, et al. Strong, Light, Multifunctional Fibers of Carbon Nanotubes with Ultrahigh Conductivity. Science. 2013;339(6116):182-6. 
[17] Zhang M, Atkinson KR, Baughman RH. Multifunctional Carbon Nanotube Yarns by Downsizing an Ancient Technology. Science. 2004;306(5700):1358-61.

[18] Koziol K, Vilatela J, Moisala A, Motta M, Cunniff P, Sennett M, et al. HighPerformance Carbon Nanotube Fiber. Science. 2007;318(5858):1892-5.

[19] Zhang M, Fang SL, Zakhidov AA, Lee SB, Aliev AE, Williams CD, et al. Strong, Transparent, Multifunctional, Carbon Nanotube Sheets. Science. 2005;309(5738):1215-9.

[20] Ma W, Song L, Yang R, Zhang T, Zhao Y, Sun L, et al. Directly Synthesized Strong, Highly Conducting, Transparent Single-Walled Carbon Nanotube Films. Nano Lett. 2007;7(8):2307-11.

[21] Endo M, Muramatsu H, Hayashi T, Kim YA, Terrones M, Dresselhaus NS. 'Buckypaper' from Coaxial Nanotubes. Nature. 2005;433(7025):476-.

[22] Hata K, Futaba DN, Mizuno K, Namai T, Yumura M, Iijima S. Water-Assisted Highly Efficient Synthesis of Impurity-Free Single-Walled Carbon Nanotubes. Science. 2004;306(5700):1362-4.

[23] Gui X, Wei J, Wang K, Cao A, Zhu H, Jia Y, et al. Carbon Nanotube Sponges. Adv Mater. 2010;22(5):617-21.

[24] Gui XC, Cao AY, Wei JQ, Li HB, Jia Y, Li Z, et al. Soft, Highly Conductive Nanotube Sponges and Composites with Controlled Compressibility. Acs Nano. 2010;4(4):2320-6.

[25] Bryning MB, Milkie DE, Islam MF, Hough LA, Kikkawa JM, Yodh AG. Carbon Nanotube Aerogels. Adv Mater. 2007;19(5):661-4.

[26] Cao AY, Dickrell PL, Sawyer WG, Ghasemi-Nejhad MN, Ajayan PM. Supercompressible foamlike carbon nanotube films. Science. 2005;310(5752):1307-10.

[27] Coleman JN, Khan U, Blau WJ, Gun'ko YK. Small but Strong: A Review of the Mechanical Properties of Carbon Nanotube-Polymer Composites. Carbon. 2006;44(9):162452.

[28] Coleman JN, Khan U, Gun'ko YK. Mechanical Reinforcement of Polymers Using Carbon Nanotubes. Adv Mater. 2006;18(6):689-706.

[29] Espinosa HD, Filleter T, Naraghi M. Multiscale Experimental Mechanics of Hierarchical Carbon-Based Materials. Adv Mater. 2012;24(21):2805-23.

[30] Filleter T, Espinosa HD. Multi-Scale Mechanical Improvement Produced in Carbon Nanotube Fibers by Irradiation Cross-Linking. Carbon. 2013;56(0):1-11.

[31] Ajayan PM, Tour JM. Materials Science - Nanotube Composites. Nature. 2007;447(7148):1066-8.

[32] Gou JH. Single-Walled Nanotube Bucky Paper and Nanocomposite. Polym Int. 2006;55(11):1283-8.

[33] Wu AS, Chou T-W. Carbon Nanotube Fibers for Advanced Composites. Materials Today. 2012;15(7-8):302-10.

[34] Lu W, Zu M, Byun J-H, Kim B-S, Chou T-W. State of the Art of Carbon Nanotube Fibers: Opportunities and Challenges. Adv Mater. 2012;24(14):1805-33.

[35] Cheng QF, Bao JW, Park J, Liang ZY, Zhang C, Wang B. High Mechanical Performance Composite Conductor: Multi-Walled Carbon Nanotube Sheet/Bismaleimide Nanocomposites. Adv Funct Mater. 2009;19(20):3219-25. 
[36] Ma WJ, Liu LQ, Zhang Z, Yang R, Liu G, Zhang TH, et al. High-Strength Composite Fibers: Realizing True Potential of Carbon Nanotubes in Polymer Matrix through Continuous Reticulate Architecture and Molecular Level Couplings. Nano Lett. 2009;9(8):2855-61.

[37] Wang X, Yong ZZ, Li QW, Bradford PD, Liu W, Tucker DS, et al. Ultrastrong, Stiff and Multifunctional Carbon Nanotube Composites. Materials Research Letters. 2012;1(1):1925 .

[38] Di JT, Hu DM, Chen HY, Yong ZZ, Chen MH, Feng ZH, et al. Ultrastrong, Foldable, and Highly Conductive Carbon Nanotube Film. Acs Nano. 2012;6(6):5457-64.

[39] Liu W, Zhang XH, Xu G, Bradford PD, Wang X, Zhao HB, et al. Producing Superior Composites by Winding Carbon Nanotubes onto a Mandrel under a Poly(vinyl alcohol) Spray. Carbon. 2011;49(14):4786-91.

[40] Banhart F. Irradiation Effects in Carbon Nanostructures. Reports on Progress in Physics. 1999;62(8):1181-221.

[41] Krasheninnikov AV, Nordlund K. Ion and Electron Irradiation-Induced Effects in Nanostructured Materials. J Appl Phys. 2010;107(7):071301.

[42] Krasheninnikov AV, Banhart F. Engineering of Nanostructured Carbon Materials with Electron or Ion Beams. Nature Materials. 2007;6(10):723-33.

[43] Kis A, Csanyi G, Salvetat JP, Lee TN, Couteau E, Kulik AJ, et al. Reinforcement of Single-Walled Carbon Nanotube Bundles by Intertube Bridging. Nature Materials. 2004;3(3):153-7.

[44] Filleter T, Bernal R, Li S, Espinosa HD. Ultrahigh Strength and Stiffness in CrossLinked Hierarchical Carbon Nanotube Bundles. Adv Mater. 2011;23(25):2855-60.

[45] Miko C, Milas M, Seo JW, Gaal R, Kulik A, Forro L. Effect of ultraviolet light irradiation on macroscopic single-walled carbon nanotube bundles. Applied Physics Letters. 2006;88(15).

[46] Ajayan PM, Banhart F. Strong Bundles. Nature Materials. 2004;3(3):135-6.

[47] Ziegler JF, Ziegler MD, Biersack JP. SRIM - The Stopping and Range of Ions in Matter (2010). Nuclear Instruments \& Methods in Physics Research Section B-Beam Interactions with Materials and Atoms. 2010;268(11-12):1818-23.

[48] Charnvanichborikarn S, Shin SJ, Worsley MA, Kucheyev SO. Tailoring Properties of Carbon-Nanotube-Based Foams by Ion Bombardment. Applied Physics Letters. 2012;101(10):103114.

[49] Shim BS, Zhu J, Jan E, Critchley K, Ho S, Podsiadlo P, et al. Multiparameter Structural Optimization of Single-Walled Carbon Nanotube Composites: Toward Record Strength, Stiffness, and Toughness. ACS Nano. 2009;3(7):1711-22.

[50] Li JZ, Gao Y, Ma WJ, Liu LQ, Zhang Z, Niu ZQ, et al. High Performance, Freestanding and Superthin Carbon Nanotube/Epoxy Nanocomposite Films. Nanoscale. 2011;3(9):3731-6.

[51] Rao AM, Chen J, Richter E, Schlecht U, Eklund PC, Haddon RC, et al. Effect of van der Waals Interactions on the Raman Modes in Single Walled Carbon Nanotubes. Phys Rev Lett. 2001;86(17):3895-8.

[52] Tirira J, Serruys Y, Trocellier P. Forward Recoil Spectrometry. New York: Plenum; 1996. 
[53] Doolittle LR. Algorithms for the Rapid Simulation of Rutherford Backscattering Spectra. Nuclear Instruments \& Methods in Physics Research Section B-Beam Interactions with Materials and Atoms. 1985;9(3):344-51.

[54] Baglin JEE, Kellock AJ, Crockett MA, Shih AH. Absolute Cross-Section for Hydrogen Forward Scattering. Nuclear Instruments \& Methods in Physics Research Section B-Beam Interactions with Materials and Atoms. 1992;64(1-4):469-74.

[55] Ziegler JF, Biersack JP, Littmark U. The Stopping and Range of Ions in Solids. New York: Pergamon Press; 1985.

[56] Song L, Ci L, Lv L, Zhou ZP, Yan XQ, Liu DF, et al. Direct Synthesis of a Macroscale Single-Walled Carbon Nanotube Non-Woven Material. Adv Mater. 2004;16(17):1529-34.

[57] Halpin JC, Kardos JL. Halpin-Tsai Equations - Review. Polym Eng Sci. 1976;16(5):344-52.

[58] Gibson LJ, Ashby MF. Cellular Solids. 2nd Edition ed. Cambridge: Cambridge University Press; 1997.

[59] Egusa S, Kirk MA, Birtcher RC. Neutron-Irradiation Effects on the MechanicalProperties of Organic Composite-Materials. J Nucl Mater. 1984;126(2):152-9.

[60] Vignoud L, David L, Sixou B, Vigier G, Stevenson I. Effect of Electron Irradiation on the Mechanical Properties of DGEBA/DDM Epoxy Resins. Nuclear Instruments \& Methods in Physics Research Section B-Beam Interactions with Materials and Atoms. 2001;185:33640 .

[61] Dresselhaus MS, Jorio A, Souza AG, Saito R. Defect Characterization in Graphene and Carbon Nanotubes Using Raman Spectroscopy. Philosophical Transactions of the Royal Society a-Mathematical Physical and Engineering Sciences. 2010;368(1932):5355-77.

[62] Ferrari AC, Robertson J. Interpretation of Raman Spectra of Disordered and Amorphous Carbon. Physical Review B. 2000;61(20):14095-107.

[63] Ferrari AC, Basko DM. Raman Spectroscopy as a Versatile Tool for Studying the Properties of Graphene. Nat Nanotechnol. 2013;8(4):235-46.

[64] Cancado LG, Jorio A, Martins Ferreira EH, Stavale F, Achete CA, Capaz RB, et al. Quantifying Defects in Graphene via Raman Spectroscopy at Different Excitation Energies. Nano Lett. 2011;11(8):3190-6.

[65] Duchamp M, Meunier R, Smajda R, Mionic M, Magrez A, Seo JW, et al. Reinforcing multiwall carbon nanotubes by electron beam irradiation. J Appl Phys. 2010;108(8).

[66] Lehtinen O, Nikitin T, Krasheninnikov AV, Sun L, Banhart F, Khriachtchev L, et al. Characterization of ion-irradiation-induced defects in multi-walled carbon nanotubes. New Journal of Physics. 2011;13.

[67] Aitkaliyeva A, Shao L. The production of amorphous regions in carbon nanotubes by $140 \mathrm{keV}$ He ion irradiation. Carbon. 2012;50(12):4680-4.

[68] Wang SR, Liang ZY, Wang B, Zhang C. High-Strength and Multifunctional Macroscopic Fabric of Single-Walled Carbon Nanotubes. Adv Mater. 2007;19(9):1257-61.

[69] Miao MH, Hawkins SC, Cai JY, Gengenbach TR, Knott R, Huynh CP. Effect of Gamma-Irradiation on the Mechanical Properties of Carbon Nanotube Yarns. Carbon. 2011;49(14):4940-7. 
[70] Gibbons JF. Ion Implantation in Semiconductors. 2. Damage Production and Annealing. Proceedings of the Institute of Electrical and Electronics Engineers. 1972;60(9):1062-96.

[71] Fonseca AF, Borders T, Baughman RH, Cho K. Load Transfer Between Cross-Linked Walls of a Carbon Nanotube. Physical Review B. 2010;81(4):045429.

[72] Byrne EM, McCarthy MA, Xia Z, Curtin WA. Multiwall Nanotubes Can Be Stronger than Single Wall Nanotubes and Implications for Nanocomposite Design. Phys Rev Lett. 2009;103(4):045502.

[73] da Silva AJR, Fazzio A, Antonelli A. Bundling Up Carbon Nanotubes through Wigner Defects. Nano Lett. 2005;5(6):1045-9.

[74] Telling RH, Ewels CP, El-Barbary AA, Heggie MI. Wigner defects bridge the graphite gap. Nature Materials. 2003;2(5):333-7.

[75] Astrom JA, Krasheninnikov AV, Nordlund K. Carbon Nanotube Mats and Fibers with Irradiation-Improved Mechanical Characteristics: A Theoretical Model. Phys Rev Lett. 2004;93(21).

[76] Astrom JA, Makinen JP, Hirvonen H, Timonen J. Stiffness of Compressed Fiber Mats. J Appl Phys. 2000;88(9):5056-61.

[77] Astrom JA, Latva-Kokko M, Kahkonen S, Makinen JP, Timonen J. The Role of Connectivity in the Properties of Sedimented Materials. Granular Matter. 2003;5(2):99-103.

[78] Cornwell CF, Welch CR. Very-High-Strength (60-GPa) Carbon Nanotube Fiber Design Based on Molecular Dynamics Simulations. J Chem Phys. 2011;134(20):204708.

[79] Naraghi M, Filleter T, Moravsky A, Locascio M, Loutfy RO, Espinosa HD. A Multiscale Study of High Performance Double-Walled Nanotube-Polymer Fibers. Acs Nano. 2010;4(11):6463-76.

[80] Rubin L, Poate J. Ion Implantation in Silicon Technology. The Industrial Physicist. 2003:12-5. 